\title{
RADIOGRAPHY OF THE PAST - THREE DIMENSIONAL, VIRTUAL RECONSTRUCTION OF A ROMAN TOWN IN LUSITANIA
}

\author{
Michael Klein a, Frank Vermeulen b, Cristina Corsi c/d,
}

a 7reasons Medien GmbH, 1200 Vienna, Bäuerlegasse 3-4, Austria, michael.klein@7reasons.at b Ghent University, Department of Archaeology, 9000 Gent, Sint-Pietersnieuwstraat 35, frank.vermeulen@UGent.be c University of Évora CIDEHUS, 7002-554 Évora, Palácio do Vimioso Apartado 94, Portugal, cricorsi@uevora.pt d University of Cassino, Department of Humanistics, 03043 Cassino (FR), Via Marconi 10, Italy, c.corsi@unicas.it

KEY WORDS: Radio-Past, Radiography, Virtual Reconstruction, 3D Modeling, Landscape Reconstruction, Roman Architecture, Lusitania

\section{ABSTRACT:}

The European project, "RADIO-PAST" was launched in 2009 within the Marie Curie framework "Industry-Academia Partnerships and Pathways". The project aims to join resources and different skills to tackle each possible aspect connected with "non-destructive" approaches to understand and reconstruct complex archaeological sites. The consortium of 7 partners has chosen an "open laboratory for research and experimentation" in and around the abandoned Roman site of Ammaia in central Portugal, but some research activities are carried out by the partner institutions in different areas of the Mediterranean and continental Europe. This paper describes the various methods and procedures which were used to undertake the three dimensional reconstruction of this Roman urban site in Lusitania.

\section{INTRODUCTION}

\subsection{Introduction}

The former Roman town of Ammaia in central Portugal serves as an ideal test site to apply state-of-the-art technology for non destructive surveys uncovering the remains of the complete settlement, due to its exceptional condition of preservation.

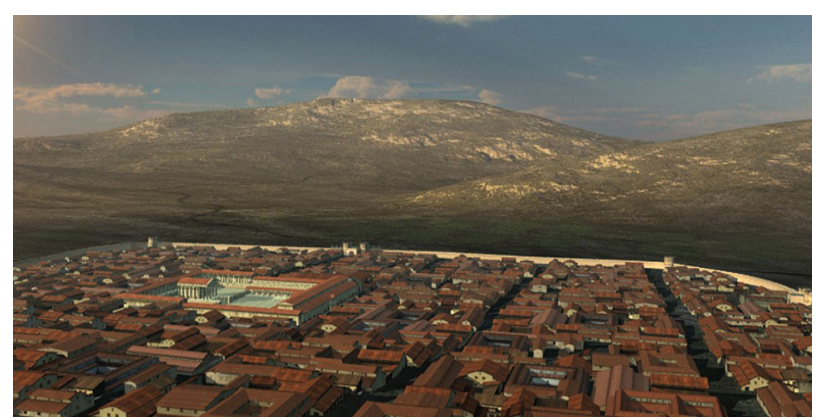

Figure 1: Birds Eye View of Ammaia

Ammaia was founded at the beginning of our era and was definitely deserted by the time of the arrival of the Arabs in the $9^{\text {th }}$ century. Its intra-mural and suburban areas are now almost free from modern construction and as the site has well preserved underground structures (stone foundations, walls, floors...) it is ideally suited for a wide range of non-invasive investigations, enlarging the database of small-scale excavations in an exponential way. Since 2000 geoarchaeological research by interdisciplinary teams of archaeologists and geomorphologists from the universities of Évora, Ghent and Cassino have achieved very intensive fieldwork here, integrating traditional excavations and ground truthing with large scale geophysical surveys, some aerial photography and GIS-based data processing. Especially since 2009, when the European Marie Curie project "Radiography of the Past" was launched and Ammaia was chosen as an open laboratory for research and experimentation, more intensive fieldwork allowed to reveal the full plan of this Roman city. Especially the evidence from geophysical survey, combined with stratigraphic and geomorphological observations, allow excellent study of the ancient urbanism, at the same time limiting the necessity for grand scale and costly excavation procedures, allowing a 3D view of the Roman townscape and opening prospects for the sustainable touristic exploitation and cultural validation of a complex site.

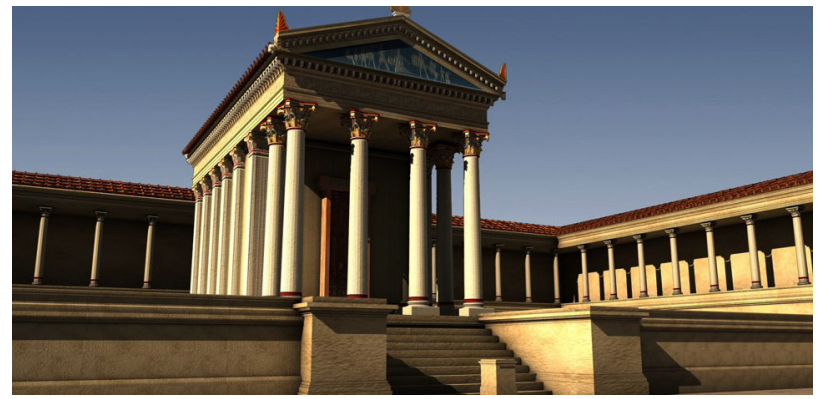

Figure 2: Forum of Ammaia

The obtained total coverage data in the form of Magnetometer and Ground Penetrating Radar Images and interpretations are used to approach a complete virtual reconstruction of the town and its surrounding landscape. The visualization of the geophysical results are approached by referencing the existing data with better preserved sites of the region comparing similar structures and dimensions, aiming to simulate local architectural local and details of decoration. The methods involved in such virtual reconstructions are multifarious and have to be adapted to the special characteristics of this site. Procedures which had been developed and tested over the past years elsewhere are being applied and refined while other new techniques had to be developed to suit the necessity of this project. The work flow as well as the main fields of activities shall be described hereafter. 


\section{WORKFLOW AND FIELDS OF ACTIVITY}

\subsection{Data acquisition}

Typically the data used during reconstruction projects is furnished by the scientific institutions involved in the project: documents, maps (old and new), pictures and images from archives, excavation reports, aerial photography, LIDAR (Light Detection and Ranging), GPR (Ground Penetrating Radar), ground magnetic survey data, etc. and their interpretations by geophysicists and archaeologists.

Further approaches of digitalisation include photogrammetric geometrisation. With the aid of current software and a series of images taken from several sides of an object the creation of accurate geometry is possible without applying markers or circumstantial measurements. This procedure can be utilized in case of original artifacts of any size as well as aerial vistas.

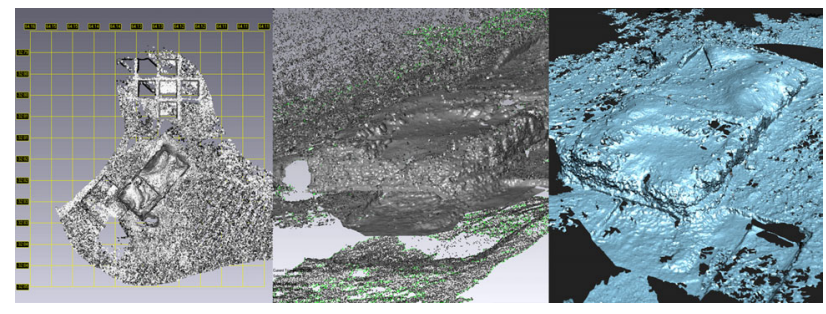

Figure 3: Laser scan of the Forum Temple

\subsection{Archiving and data pre-processing}

As part of the archiving activity a first processing of raw data from photogrammetric surveys (images) and scans (3D point clouds) is achieved. To acquire high-quality $3 \mathrm{D}$ models for further steps in reconstruction projects, raw survey data is processed with different programs; the extraction of textured models with different geometric resolution is usually one of the main issues to decide, depending on the environment where the model is used or placed. One special procedure was established to suit the need of a large online database, where highresolution scanned objects had to be reduced to $1 \%$ of their original density, while still preserving all of their visual qualities.

\subsection{Archaeological databases}

Due to the fact that several archives started to digitalize their archaeological finds, the need to present this data in an ordered and accessible way to a broader research community and the public is increasing. The scanned objects are displayed on the Internet. To display this media on various platforms, viewers like Adobe flash, 3D-PDF or html 5 are used. Previews of these objects are presented in a lightweight format to make them accessible to all hardware devices.

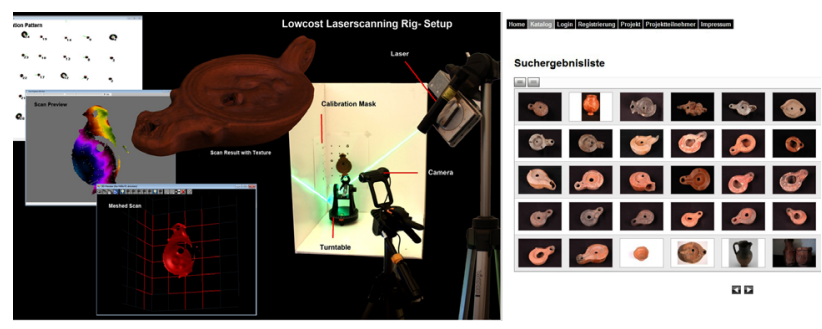

Figure 4: Data Acquisition and final result of a 3d Database
Accurate copies of the scanned data can be accessed by registration in the database and after a download used for ongoing research. By developing a special procedure we are now capable of processing great amounts of items within comparably short time. This digital archiving activity was started in 2011 for the huge database of objects present in the archaeological laboratory and museum of Ammaia. Although meant as a research instrument, part of the scanned material, consisting essentially of well preserved objects such as stelae, architectural decoration, pottery, lamps, glassware, etc., can be of great use for the virtual town reconstruction.

\subsection{GIS - data integration}

To combine data used in large-scale reconstruction like the remodeling of urban settlements and towns, GIS systems are being used. Various georeferenced cartographic and topographic information was collected and assembled to construct a visual database which is used in the particular task of reconstruction. Topographical data like DHM (Digital Elevation Model) and DTM (Digital Terrain Model) is needed for the generation of the virtual environment, which is further modified to achieve an approximation to the state of the desired period. To accomplish these tasks specialised terrain simulators and editors are used to calculate processes of erosion and flow of water. The results are displayed in a fractal geometry, producing a highly realistic and natural appearance. A further task is the insertion of historical maps which can be used to approximate various historical landscape features like river courses, streets and settlement boundaries, including some of the archaeological and geomorphological field data merged via expert knowledge present in the multidisciplinary team. In this way a first base for a visualisation model of the terrain and landscape around Ammaia was achieved. This model needs to be further refined in the future once more data, such as from palaeo-ecological and additional geomorphological research, are available.

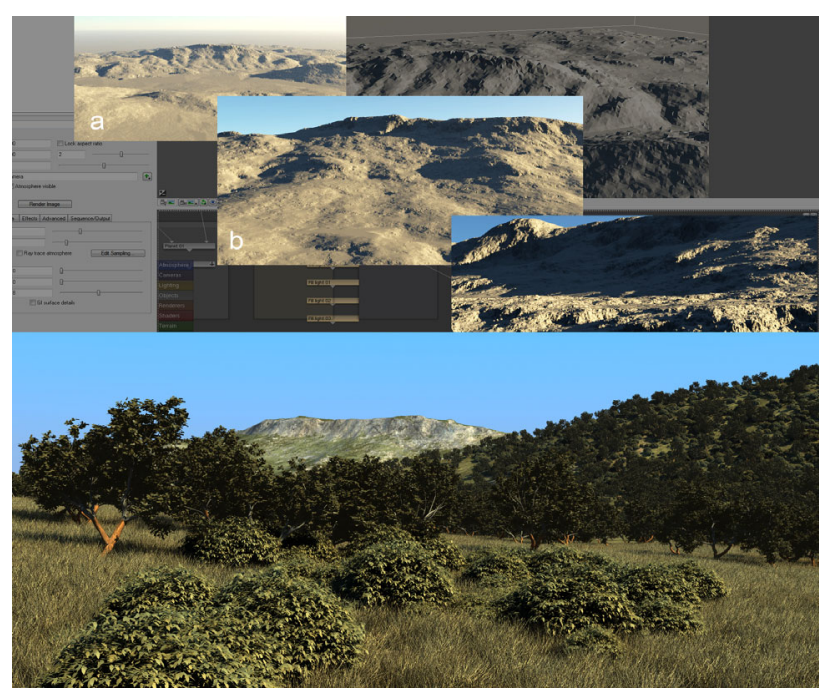

Figure 5: Digital Terrain in various stages of the editing process

\subsection{Process of reconstruction}

Cultural landscape, buildings and artefacts are digitally recreated or their scan incorporated into virtual environments to present them in their presumed original context. Through constant consultation and discussion between the virtual 
reconstruction team and the archaeologists authenticity and correctness of the models is ensured. With the help of excavation plans, building remains and unearthed artefacts, the development of comprehensive models of ancient life is sought. The presentation of towns or smaller settlements and the use of the surrounding environment by their inhabitants, building typology for representative buildings as well as for vernacular architecture is recreated. To increase the detail of singular buildings and complexes and to show functionality of interior space finds of the site are displayed at their presumed position. Very often the finds of one particular archaeological site are not sufficient enough to allow for a comprehensive reconstruction. In this case data has to be obtained by comparative research, by using information from sites of the same period, geographical region and importance. For the site of Ammaia, the Lusitanian context and in particular data from well excavated parts of the provincial capital Augusta Emerita and the extensively studied site of Conimbriga are of particular relevance, as are in more general terms data pertaining to excavation sites from all over the Iberian peninsula. To understand questions of operating cycles, effectiveness and the correct use of ancient tools and certain Roman machinery (pumps, lifting devices...), functional presentations are devised and simulations carried out.

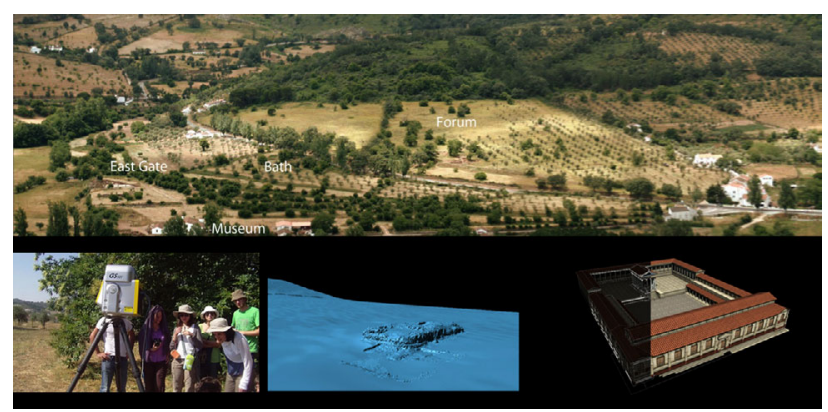

Figure 6: Digitalization of the Forums Temple and a possible Visualisation

\section{METHODS}

Through several sessions involving archaeologists, historians and other researchers the outline of the project is determined: what scope the reconstruction should have, what kind of material is accessible and which form the final presentation will take. After viewing the material, the first approaches are developed: in case of settlement reconstructions a low resolution model is drawn with settlement boundaries, streets, the space occupied by buildings, etc . Usually also the delineation of public areas with representative buildings, such as the forum and public baths (which are typically better documented) and the rest of the settlement occurs. Also very helpful is the fact, that in the past most architecture, especially houses were built complying to a comparatively uniform code of design, presenting a common tradition of a social class or of a population. To determine these building types archaeologists supply excavation results, images of still existing built examples or historical descriptions and drawings. Also specially designated areas within a settlement, like gardens with outbuildings or fortifications, have to be marked out and drawn in $3 \mathrm{D}$. At this point already a crude $3 \mathrm{D}$ model of the settlement exists, which is inserted into a realtime engine, to allow modification and interactive display during further sessions with the archaeologists. This point is crucial in every project, as several questions remain characteristically unsolved, and can be studied in detail with the help of the model and incorporated data like maps, the terrain model and excavation plans. When satisfactory answers have been found, and the layout thus modified, the houses in the scene are textured, to give them a realistic appearance and also the landscape, roads and other features are modeled more in detail. Sometimes the decision is made to show a close-up of certain objects, like a single house or compound. In this case a model with higher degree of geometric resolution has to be created, showing also the interior organisation, like rooms, stairs and corridors. To complete the scene, correct building materials, interior and furniture have to be shown. This is a task which is of similar importance as the quality of the general layout, if the overall result should be scientifically correct. In the case of a short film format, a camera path is created to define the sceneries. According to the camera's clipping, scenes can be further refined and a storyline can be established. To give a reference to human proportions and to provide a more interesting backdrop, animated human figures and artifacts characteristic for the given time are inserted. On the other hand the material can be used to produce interactive $3 \mathrm{D}$ real-time applications, allowing the user to freely move around the scenes and access information on demand. This is particularly relevant here in Ammaia as the site has its own on-site museum.

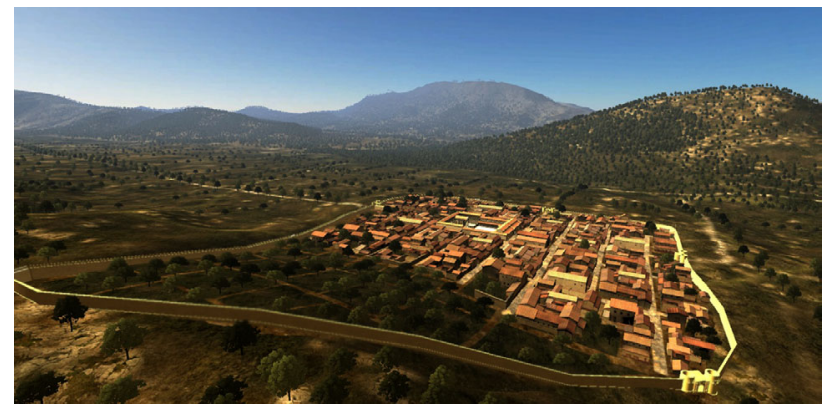

Figure 7: Sketch of Ammaia within a 3D realtime environment

\section{TECHNIQUES}

\subsection{Modeling and texturing of objects}

To sketch out ideas for a reconstructed scene a coarse geometry is laid out to be presented and discussed with the scientific partners. Various methods of production are implemented depending on the use for the final format. Accuracy and authenticity is crucial to obtain a high quality, together with the documentation of the resulting objects. When the desired level of detail is achieved, the process of texturing (mapping images onto the surfaces) is applied.

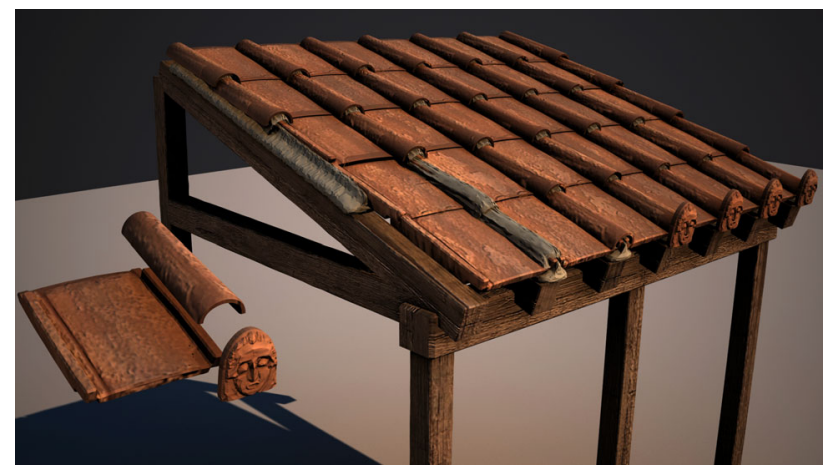

Figure 8: Detailed visualisation of roman roofing 
A wide range of software is used for the construction of different items and demands highly skilled personal to ensure a high quality output.

\subsection{Landscape visualisation}

Incoming data from GIS applications were transformed according to the needs to achieve a historical accurate simulation based on manually driven construction methods. The best available technology to output a highly realistic terrain is a fractal approach. The detail of the geometry is adapted depending on the distance to the virtual camera. With this method replicas of millions of items (boulders, trees, houses, etc.) can be spread over the landscape. Certain terrain features like streets, urban boundaries, agricultural land-use or waterways can be defined by simple maps possessing RGB and gray scale values, which are called splat-maps. The ease of use of these splat-maps is one of their advantages, allowing to assemble the information as a simple image which can be edited by the non computer-graphic specialist members of the project team.

If available, LIDAR data can also be used to filter and discard various recent features like vegetation growth and modern construction, allowing in some cases the detection of archaeological remains. Apart from the archaeological interpretation of data from geophysics survey and excavation, aerial images taken from airplanes were used to build geometry without the cost-intensive and laborious procedure of a LIDAR scan with the use of photogrammetry. Fluid simulation was applied to visualise realistic water and simulate river flows of the river Sever and its tributaries.

As no high resolution LIDAR coverage data were available for the Ammaia-site we had to make use of a dense DGPS survey which revealed not only the current microtopography in sufficient detail, but eventually helped in filtering out modern elements in the landscape, such as current vegetation and agricultural terracing. Together with the geomorphological survey data they provided a crucial base for the simulation of the early Imperial Roman topography of the area, easily including all elements of standing architecture visible above ground today, such as parts of the city defenses and excavated building structures.

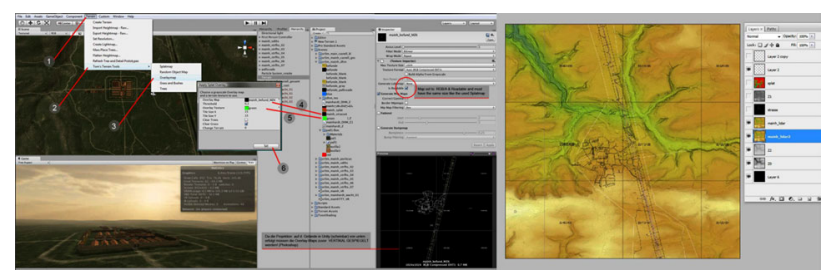

Figure 9: Topographic data editing environment

\subsection{Character creation and animation}

Still in process, as part of the visualisation of early Imperial Ammaia, is the insertion of characters and animations. Humanoid and non-humanoid 3D- models are being created with a toolset specialised for organic construction. Depending on the needs this process can start up from a skeletal phase building up volume by applying muscular systems and ending at the texturing process.

When the desired shape of a model is reached, a kinematic bone structure is inserted with a mesh-binding which allows animation of various parts to proceed. The results can be used for highly detailed visuals as well as crowd animations to enliven reconstructed sceneries, adapted here to the specifics of a Roman or Romanized town and suburban population with a strong link with the countryside and its exploitation.

\subsection{Motion-capture}

The in-house motion-capture system at 7reasons consists of a magnetic suit, which allows an actor to perform freely in every environment. This can be used to drive a virtual character constraining him to the movement of a specialist or an actor performing in this suit. Optical systems are used to reproduce facial and complete body movements as well as gesture recognition. Data cleaning and - preparation must be done in conjunction with the recording to assure smooth transitions in movement and behaviour. Complete capture sessions can be applied but also smaller parts of these sessions can be used to create clips which then are utilised in turn to create a storyline similar to common film editing. These systems are applied to characters within 3D sceneries for movie production and realtime applications of Early Roman Imperial life in Ammaia.

\section{DOCUMENTATION}

The decisions taken during the reconstruction process are documented and commented in online blogs, citing used material, comparative sources etc. This ensures not only a good communication within the project team, but also transparency, retraceability and a scholarly approach to the topics in question. With this proper base the evolution of reconstruction concepts can be published later on in scientific papers. Without these measures most of the work conducted would be rendered meaningless, as the main goal cannot only be to produce attractive images of historic objects and scenery, but to gain new insights and knowledge.

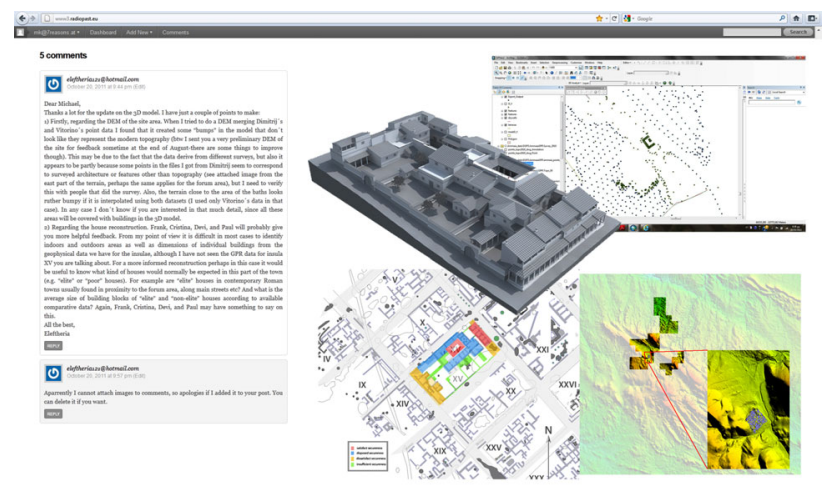

Figure 10: Blog discussion of a suggested reconstruction of Insula X

\section{OUTPUT}

\subsection{Realtime applications}

Realtime applications are made for almost all platforms and hardware devices delivering interactive scenery to the spectator inviting him to discover reconstructed sites and information. The long lasting experience of 7reasons in these fields enables us to apply innovative solutions like archaeological sandboxsystems to aid communication between scientists and artists. This principle can also be used to transfer ideas of a project team to a broader audience. The increasing quality of the current game engines will allow us to produce, in the near future, not only realtime but also film footage without the need 
of rendering. Various productions have been made recently handling large data sets, such as in the project of the GermanRaetian limes, where over $170 \mathrm{~km}$ of scanned terrain data was inserted. Other successful productions like those about Carnuntum, Marvao, Caerleon and the Austrian limes show that the new trend of realtime media is very well received by the market.

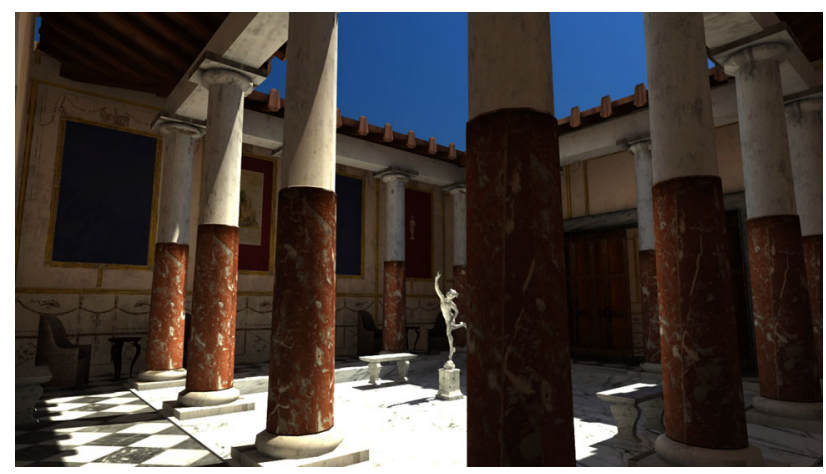

Figure 11: Realtime Walkthrough of a roman Villa

\subsection{Short film production}

The classical short film production will always be a major part of the media market due to its advantage of linear storytelling. To produce hundreds of thousands of images which are assembled and edited in the post-production phase, we use a considerable number of computers combined to a render-farm.

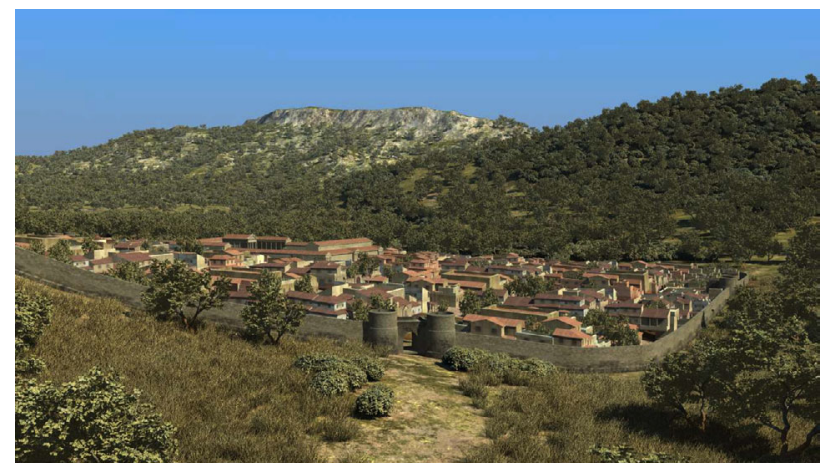

Figure 12: Screenshot from a test sequence for the planned short film of Ammaia

\section{CONCLUSION}

A majority of the explained procedures and techniques have been applied to the Ammaia project resulting in the first images and models of the ancient landscape setting and large parts of the Roman city. The commitment to create all the necessary visualizations can only be fulfilled with the complementation of the scientific data and their interpretation, which is currently in a final stage. Therefore, the main task of the total reconstruction is still ahead and will be processed within 2012. Many new approaches like the "Three dimensional modular construction system" for interpretation and visualization of archaeological urban environments have been developed and integrated, but also other methods and tools used in former projects could be successfully verified and applied within this Ammaia project. The experience gained throughout this work was tremendous and we will gladly share this empirical knowledge in the future.

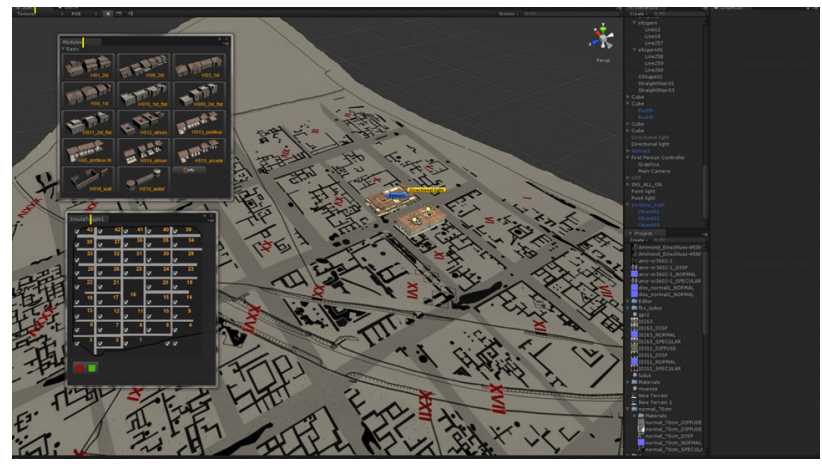

Figure 12: Realtime editor with drag and drop library of building - block modules

References from Proceedings and Books:

Corsi, C., Klein, M. Weinlinger, G.: The Roman town of Ammaia (Portugal): From total survey to 3D reconstruction. In: Proceedings of the $16^{\text {th }}$ CHDT Meeting - Vienna 2011(forthcoming).

Humer, F., Gugl, C., Pregesbauer, M., Vermeulen, F., Corsi, C., Klein, M.: Current Productions Carnuntum, German Limes and Radiopast, Virtual Archaeology Review, 4 (http://varjournal.es/doc/varj02_004_16.pdf) (2011).

Van Roode, S., Vermeulen, F., Corsi, C., Klein, M., Weinlinger, G.: Radiography of a townscape. Understanding, visualising and managing a Roman townsite, Proceedings of the congress $L A C$ 2010, Amsterdam, 429-441 (2012).

Vermeulen, F., Corsi, C., De Dapper, M.: Surveying the townscape of Roman Ammaia (Alentejo, Portugal): a geoarchaeological approach of the forum area, Geoarchaeology, vol. 27( 2), 105-187 (2012).

Vermeulen, F., Taelman, D.: From cityscape to landscape in Roman Lusitania: the municipium of Ammaia. In: Corsi, C., Vermeulen, F. (eds.), Changing Landscapes. The impact of Roman towns in the Western Mediterranean (Proceedings of the International Colloquium, Castelo de Vide - Marvão 15th-17th May 2008), Bologna, 2010, 311-324 (2010).

\section{References from websites:}

http://www.radiopast.eu, http://www.7reasons.at

\subsection{Acknowledgements and Appendix}

Project Partners: Being an acronym of Radiography of the Past, Radio-Past (2009-2013) concerns a project of the "family" People - Marie Curie Action IAPP (Industry-Academia Partnerships and Pathways), where 4 academic and 3 industrial partners join their resources to develop integrated nondestructive approaches to understand and valorise complex archaeological sites. These partners are: Universidade de Évora (Portugal), Universiteit Gent (Belgium), Univerza v Ljubljani (Slovenia), 7reasons Media (Austria), British School at Rome (United Kingdom), Past2Present (Netherlands), Eastern Atlas (Germany). 
